\title{
Quantifying Nanomolar Protein Concentrations Using Designed DNA Carriers and Solid-State Nanopores
}

\author{
Jinglin Kong, Nicholas A. W. Bell, and Ulrich F. Keyser* \\ Cavendish Laboratory, University of Cambridge, JJ Thomson Ave, Cambridge, CB3 0HE, United Kingdom
}

Supporting Information

ABSTRACT: Designed "DNA carriers" have been proposed as a new method for nanopore based specific protein detection. In this system, target protein molecules bind to a long DNA strand at a defined position creating a second level transient current drop against the background DNA translocation. Here, we demonstrate the ability of this system to quantify protein concentrations in the nanomolar range. After incubation with target protein at different concentrations, the fraction of DNA translocations showing a secondary current spike allows for the quantification of the corresponding protein concentration. For our proof-of-principle experiments we use two standard binding systems, biotin-streptavidin and

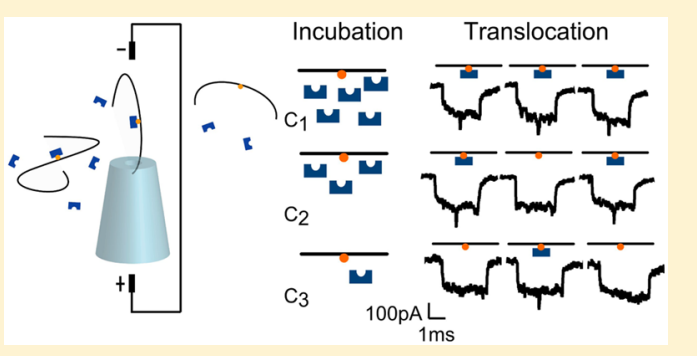
digoxigenin-antidigoxigenin, that allow for measurements of the concentration down to the low nanomolar range. The results demonstrate the potential for a novel quantitative and specific protein detection scheme using the DNA carrier method.

KEYWORDS: Nanopore, proteins, quantifying, DNA, concentrations, biosensing

$\mathrm{P}$ roteins are essential for living systems and serve in diverse roles for instance in transport, catalysis, molecular motion and structural support. Most of their functions are modulated and controlled by both the identity and abundance. The accurate and specific measurement of protein concentrations is therefore of fundamental importance in biological and medical research, drug screening, and disease diagnosis. Several wellestablished methods are used for determining protein concentration. High accuracy can be achieved using radio labeling ${ }^{1}$ and mass spectrometry ${ }^{2}$ in which protein molecules are analyzed at peptide or single amino acid level. Specific binding based methods such as electrophoretic mobility shift (EMSA), ${ }^{3}$ fluorescent polarization (FP), ${ }^{4}$ and enzyme-linked immunosorbent assays (ELISA) ${ }^{5}$ are commonly used for routine protein concentration determination. Efforts have been made toward lowering the detection limit, required sample volumes and throughput by introducing micro or nano arrays $^{6}$ and nanoparticles.

Solid-state nanopore based sensing is being actively investigated as a versatile platform for single-molecule protein sensing. This research is driven by the potential for fast detection, avoiding the sometimes cumbersome fluorescent labeling of proteins, with a single molecule sensing method which can be integrated into silicon electronics. Furthermore, since the throughput of the acquired signal is independent of the sample volume, and rather depends on the concentration, there is the possibility for microfluidic integration ${ }^{8}$ which provides an advantage over established techniques such as ELISA and radio-labeling. However, results to-date using solidstate nanopore indicate that the translocation speeds of most protein monomers are too fast for all translocations to be accurately recorded at typical experimental bandwidths.'
Although high bandwidth systems combined with thin membranes can record most translocations in a limited voltage range, the fast translocation aspect makes it particularly challenging to accurately determine protein concentration. ${ }^{10}$ Another complication is added by the potential for various protein-nanopore wall interactions ${ }^{11}$ limiting the ability to differentiate protein species. Creating a binding site for a target protein on a long DNA strand (DNA carrier) and measuring the translocation signals of the complex helps to address these problems in protein monomer only translocations. ${ }^{12}$ First the binding site can be selective for a certain protein which enables its identification. Second the easily discernible signal from the current event produced by the DNA strand provides a marker for predicting when the protein signal will occur. Therefore, protein signals are only analyzed when occurring during the DNA signals rather than the whole experimental current trace.

In this study we employ the method of DNA carriers for evaluating protein concentration (Figure 1). We determine the concentration by measuring the fraction of DNA carriers which show a secondary signal indicating the presence of a protein. Two model systems are investigated, namely, biotinstreptavidin and digoxigenin-antidigoxigenin. Binding curves are constructed by varying the protein concentration in these two systems. Our results show good agreement between the nanopore based binding curve and a model using a dissociation constant value determined by other standard assays. For the biotin-streptavidin system, we measure a small deviation from

Received: February 12, 2016

Revised: April 26, 2016

Published: April 28, 2016 
(a)

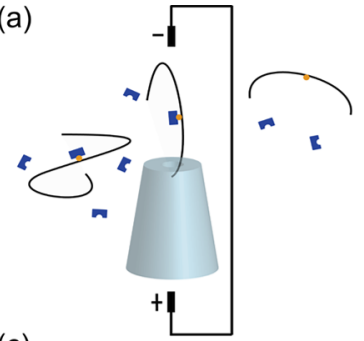

(c)

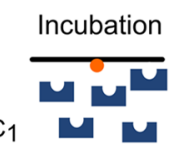

(d)

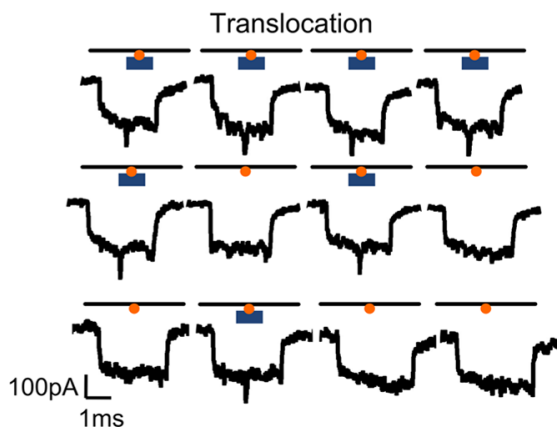

Figure 1. (a) Schematic representation of incubated DNA carrier (black line) and protein complexes (black line with a blue cuboid attached) translocating through a nanopore driven by the electric field. (b) Example occupied carrier translocation event shows an extra second level current drop in the very middle of the event, which is caused by the targeted protein binding. To find the positive events through data processing, we set a searching window of $25 \%$ of the total event duration. (c, d, e) Schematic and example DNA carrier translocation events after incubation with targeted protein of different concentrations. The concentration ratios $\left(c_{\text {Protein }} / c_{\text {Carrier }}\right)$ are decreasing from $\mathrm{c}$ to e. The carriers are fully occupied in $\mathrm{c}$ showing that all the carrier translocation events have secondary current spike positioned in the middle (occupied events), while in $\mathrm{d}$ and e, less occupied events are found. Example events are produced by the biotin-streptavidin system.

the expected binding curve which we assign to the lower signalto-noise ratio for the $52.8 \mathrm{kDa}$ streptavidin bound on the DNA.

The basic structure of the DNA carrier is fabricated by using a linearized $7.2 \mathrm{~kb}$ m13mp18 virus genome (New England BioLabs) and 190 designed oligonucleotides (Integrated DNA Technologies) each 38 bases in length. These oligonucleotides hybridize to the m13mp18 single-stranded (ss) DNA forming a long double-strand. The oligonucleotide in the center of the DNA carrier was modified with a $5^{\prime}$ extension of three thymines and either digoxigenin or biotin. Full details of the assembly were described previously. ${ }^{12}$

We use glass nanopores derived from the laser-assisted pulling of quartz glass capillaries with outer diameters of 0.5 $\mathrm{mm}$ and inner diameter of $0.2 \mathrm{~mm}$ (Sutter Instruments, USA). In order to optimize the signal-to-noise ratio, two slightly different pulling programs were used for the two different protein systems. The detailed parameters for nanopore fabrication can be found in the Supporting Information (Table S1). The magnitude of the dsDNA current drops is $-0.160 \pm 0.023 \mathrm{nA}$ and $-0.146 \pm 0.025 \mathrm{nA}$ for pulling program 1 and 2, respectively (distributions shown in Supporting Information, Figure S1, errors are standard deviations). Slightly larger nanopores produced by program 2 were used for the digoxigenin system with the aim of reducing unspecific protein-nanopore surface interactions. The dsDNA current level was used in the data analysis protocol for setting the appropriate threshold (see Supporting Information, Figure S2).

Monovalent streptavidin was generously provided by the Howarth Lab. ${ }^{13}$ The three digoxigenin antibody samples were purchased from three companies (Roche, Invitrogen, and Abcam) (monoclonal, isotype IgG). The protein concentrations were checked by measuring absorbance at $280 \mathrm{~nm}$ (Nanodrop 2000) before any dilutions were done. DNA carriers with $3 \mathrm{nM}$ concentration were incubated with the target proteins for $30 \mathrm{~min}$ in $100 \mathrm{mM} \mathrm{NaCl}$ and $2 \mathrm{mM} \mathrm{MgCl}_{2}$ buffered with $10 \mathrm{mM}$ Tris- $\mathrm{HCl}(\mathrm{pH} \sim 8)$. The sample was then diluted by adding the same volume of $8 \mathrm{M} \mathrm{LiCl}, 100 \mathrm{mM} \mathrm{NaCl}$, and $2 \mathrm{mM} \mathrm{MgCl}_{2}$ with the same buffer condition, making the final DNA carrier concentration of $1.5 \mathrm{nM}$ in $4 \mathrm{M} \mathrm{LiCl}, 100$ $\mathrm{mM} \mathrm{NaCl}$, and $2 \mathrm{mM} \mathrm{MgCl} 2$ buffered with $10 \mathrm{mM}$ Tris- $\mathrm{HCl}$ $(\mathrm{pH} \sim 7.5)$. The protein concentrations used range from 0 to $5.6 \mathrm{nM}$ for streptavidin and 0 to $31 \mathrm{nM}$ for the digoxigenin antibody. All translocation measurements were carried out at $600 \mathrm{mV}$ applied voltage. The ionic current data was acquired using an Axopatch $200 \mathrm{~B}$ with a sampling frequency of $250 \mathrm{kHz}$ and filtered at $49.9 \mathrm{kHz}$ with an external 8-pole Bessel filter.

For the independent determination of dissociation constants $\left(K_{\mathrm{d}}\right)$, electrophoretic mobility shift assay (EMSA) and fluorescence polarization (FP) were used to investigate the affinity of these two standard pairs. Detailed information on these assays is given in the Supporting Information.

The raw ionic current data contain translocations from free protein, folded DNA carriers (observed in wide diameter nanopores as those used here), and unfolded DNA carriers which pass through in a head-to-tail fashion. There are also fragments of the full DNA carrier length due to the synthesis procedure. It is important to note that we filter the translocations to remove all subpopulations except the full length, unfolded DNA carriers. This is achieved by an ECD (Event Charge Deficit) threshold and ionic current threshold (1.5 times current drop amplitude corresponding to doublestranded DNA) at the beginning and end of the events. Details of this process were described previously. ${ }^{12}$

An example translocation event after these filtering steps is shown in Figure 1b. A searching window of $25 \%$ of the event duration is created in the middle of each translocation to test for the presence of a secondary peak due to the bound protein. The threshold to find the peak was defined with a factor (I/ $\left.I_{\mathrm{DNA}}\right)$ based on the dsDNA current amplitude. The fraction of occupied events decreases with the increasing threshold factor (Supporting Information, Figure S2). We chose to use a threshold of 1.4 times the magnitude over the DNA carrier signal, $I_{\mathrm{DNA}}$, for all the data analysis in this work. This empirical threshold provides a clear differentiation between the protein binding peak and the false positive peak observed in DNA carrier measurements where there is no protein present.

We chose the biotin-streptavidin interaction as it is one of the strongest known noncovalent interactions with a dissociation constant of $K_{\mathrm{d}} \sim 10^{-15} \mathrm{M}$. Due to its extremely high affinity, this interaction is an ideal model system for determining the ability of measuring protein concentrations in the nanomolar range. First, we incubated an approximately four times stoichiometric excess of monovalent streptavidin with the DNA carrier. A few typical example events of the occupied biotin DNA carrier are shown in Figure 2a. An extra transient current drop, representing a protein binding, can be found in each of the example traces and is positioned in the middle of the observation window of each event. A histogram of the peak 
(a)

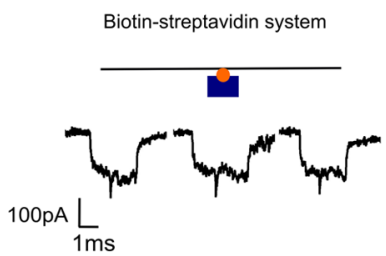

(c)

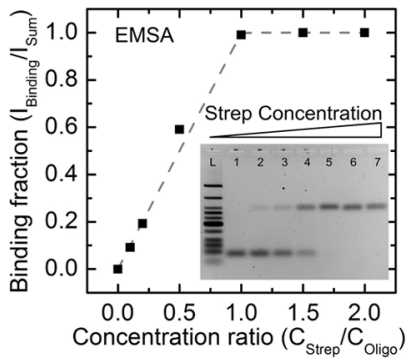

(b)
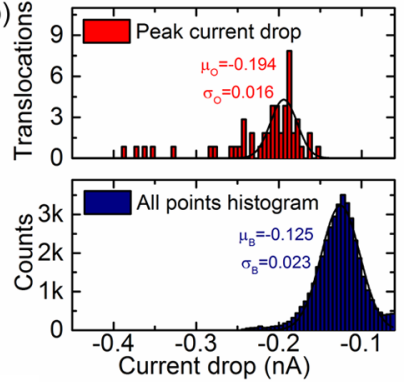

(d) Streptavidin concentration (nM)

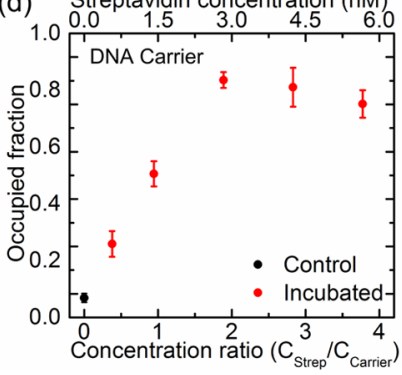

Figure 2. Concentration measurement of streptavidin using DNA carriers. (a) Schematic representation of biotin-streptavidin systems and examples of occupied DNA carrier translocation events. The monovalent streptavidin (blue cuboid) is $52.8 \mathrm{kDa}$. (b) Histogram of the peak current drop (red) and an all points histogram (blue) of all linear translocation events. The histograms are fitted by a Gaussian function, where the main peak in the all point histogram $\left(\mu_{\mathrm{B}}\right)$ determines the dsDNA level and the center of the peak current drop $\left(\mu_{\mathrm{O}}\right)$ shows the protein binding signal. The concentration ratio was 3.8 $\left(c_{\text {Streptavidin }} / \mathcal{c}_{\text {Carrier }}\right), c_{\text {Streptavidin }}=5.8 \mathrm{nM}$. (c) EMSA showing titration of monovalent streptavidin against a biotin labeled DNA duplex of $38 \mathrm{bp}$. The corresponding gel image is shown in the inset. The intensity of the lanes is determined using ImageJ. Lane $\mathrm{L}$ is a DNA ladder reference (low molecular weight from $25 \mathrm{bp}$ to $766 \mathrm{bp}$ ). The concentration of the DNA duplex was $80 \mathrm{nM}$ for each lane. Lanes 1-7 correspond to biotin labeled oligo incubated with streptavidin of different concentration ratio $\left(c_{\mathcal{S}_{\text {Streptavidin }}} / \mathcal{c}_{\text {Oligo }}\right)$ of $0,0.1,0.2,0.5,1,1.5$, and 2 , respectively. The gray dashed line shows the linear increase expected for an infinitely high affinity pairing at equilibrium. (d) Binding curve based on nanopore measurements. A DNA carrier of 1.5 $\mathrm{nM}$ concentration was incubated with monovalent protein from $0 \mathrm{nM}$ to $5.6 \mathrm{nM}$. Error bars are standard error of the mean from different nanopores. The binding curve is obtained from a total of $n=70$ nanopore experiments. The event numbers in the plots are 434, 479, $2044,689,174$, and 905 for data points at the concentration ratio from 0 to 3.8 , respectively (statistics in Supporting Information S7).

current drop (red) and an all points histogram (blue) of all linear translocation events are shown in Figure $2 b$. The two peaks in the histograms are overlapping indicating that the level of current drop caused by the monovalent streptavidin is relatively close to that of the dsDNA level. This explains why some streptavidin translocations may go undetected due to the required threshold for event detection. Biotin-streptavidin is known to retain its high affinity in a wide range of conditions and extremes of $\mathrm{pH}$, temperature and detergents ${ }^{14}$ are needed to break the interaction. Therefore, we can safely assume that the affinity remains significantly high, below subnanomolar even in the high salt solution used for nanopore experiments. Thus, we expect to observe a linear increase in the occupied fraction of DNA carriers as a function of the streptavidin concentration until all DNA carriers are saturated. As a control, EMSA was used to measure the fraction of biotinylated DNA

bound as a function of streptavidin concentration. A $38 \mathrm{bp}$ DNA duplex was synthesized with a three thymine and biotin label and the protein was titrated against this duplex. The upper bands in the gel image (Figure 2c) correspond to the duplexes with bound streptavidin while the lower bands are the unbound duplexes. The binding fraction obtained from the gel band intensity is plotted in Figure 2c. A good agreement is found between the expected linear increase (gray dashed line) and the gel intensity data.

Figure $2 \mathrm{~d}$ depicts the fraction of occupied events as a function of the concentration ratio $\left(c_{\text {Streptavidin }} / \mathcal{c}_{\text {Carrier }}\right)$ for the nanopore experiments. The lower limit is $\sim 0.1$ which is due to the false positive rate of protein-like signals observed on bare DNA. This false positive can be caused by the presence of folds or knots in the DNA or other factors. As expected, a linear increase of occupied fraction is observed until the curve levels off at a fraction of 0.8 . This leveling off at 0.8 instead of 1 is due to the signal-to-noise ratio of the streptavidin system. Some protein events are buried in the noise-as suggested by Figure $2 \mathrm{~b}$. The peak current histogram for streptavidin detection (Figure 2b, top) overlaps the current distribution of the dsDNA level (Figure 2b, bottom). Additionally, the occupied fraction saturated at a concentration ratio of 2 rather than 1 . The continuing increase beyond the expected ratio of 1 is likely due to nonspecific adsorption to walls of the fluidic chip. This is a known problem in microfluidic chips which is particularly prevalent at the nanomolar concentrations used here. ${ }^{15}$ We note that each point in Figure $2 \mathrm{~d}$ shows an average of 6-25 independent nanopore measurements and 2 or 3 different protein sample dilution runs. We measure significant variations in the occupied fraction during these repeats which are explained by signal size variations from different nanopores and the variability in nonspecific adsorption during the protein handling at nanomolar concentration (Supporting Information, Table S3).

In a second demonstration of this method, we investigated how the occupied carrier fraction varies with a digoxigeninantidigoxigenin (Anti-dig) system. The molecular weight of antidigoxigenin is $150 \mathrm{kDa}$-significantly bigger than streptavidin at $52.8 \mathrm{kDa}$. Indeed, the peak current amplitude of the occupied carrier is typically bigger than streptavidin (Figure 3a) which improves the signal-to-noise ratio and thus our detection accuracy. The improved resolution can also be directly observed in Figure $3 b$, in which the overlap between dsDNA level and protein binding level is almost nonexistent compared to the data in Figure $2 \mathrm{~b}$.

The dissociation constant for antidigoxigenin is in the range of $\mathrm{nM}$ at physiological salt concentrations. ${ }^{16}$ Here, we measured the dissociation constant of the monoclonal antidigoxigenin by fluorescence polarization. A $K_{\mathrm{d}}$ of $\sim 3.5$ $\mathrm{nM}$ was estimated in buffers with and without $4 \mathrm{M} \mathrm{LiCl}$ which is used in the nanopore experiments (see Supplementary Figure S4). Details of the fitting model are described in Supporting Information S4.

As shown in Figure 3c, the upper limit of the occupied carrier fraction is $\sim 0.95$ which is considerably higher than that of the biotin-streptavidin system $(\sim 0.8)$. This clear improvement is due to the higher signal-to-noise ratio of this larger antibody system and confirms our assertion that some streptavidinbiotin carrier translocations have a current value for the streptavidin level which is not high enough above the noise for detection. The small remaining fraction which does not show a positive signal for antidigoxigenin is due to a small fraction of 
(a)

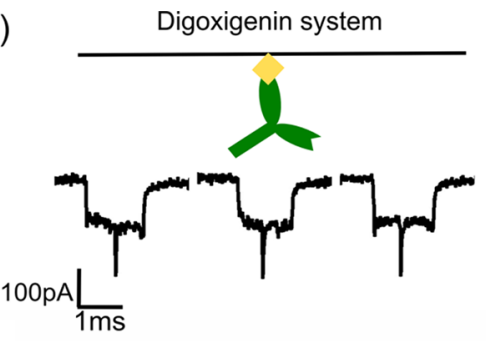

(b)

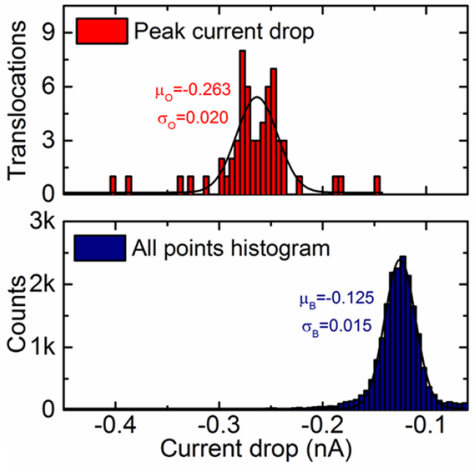

(c)

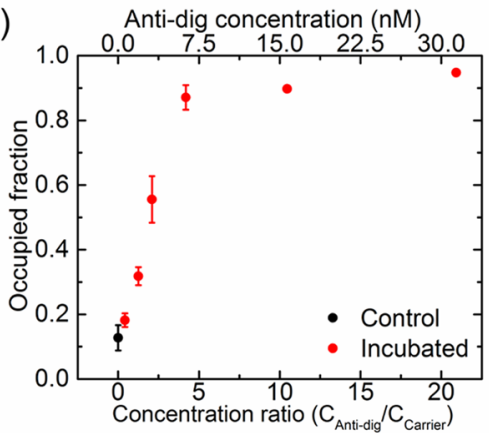

Figure 3. Concentration measurement of antidigoxigenin using DNA carriers. (a) Schematic representation of digoxigenin-antidigoxigenin system and examples of occupied digoxigenin carrier translocation events. The digoxigenin antibody (green Y-shaped structure) is about $150 \mathrm{kDa}$. (b) Histogram of the peak current drop (red) together with an all points histogram (blue) of all linear translocation events. The histograms are fitted by a Gaussians function. The concentration ratio was $10.5\left(c_{\text {Anti-dig }} / c_{\text {Carrier }}\right), c_{\text {Anti-dig }}=15.8 \mathrm{nM}$. (c) Binding curve based on nanopore measurements. A DNA carrier concentration of $1.5 \mathrm{nM}$ was incubated with monovalent protein from $0 \mathrm{nM}$ to $31 \mathrm{nM}$. Error bars are standard errors of the mean (not included for data points with two nanopores at the concentration ratio of 10.5 and 20.9). The plot represents data are from $n=25$ nanopores. The event numbers in the plots are 94,141,159, 145, 111, 93, and 32 for data points at the concentration ratio from 0 to 21 respectively (statistics in Supporting Information S7).

imperfect DNA carriers whereby not all contain the digoxigenin labeled oligonucleotide.

It has to be noted that influencing factors mentioned previously with the biotin system also apply here. Protein concentration variations among different dilutions can occur due to losses caused by nonspecific surface binding. Also, in particular for antibodies, our specific protein detection method is sensitive for the active antibodies in the sample which can therefore be different from the concentrations determined spectrophotometrically.

In order to highlight the fact that we can assess active antibody concentrations, we decided to compare binding curves of three antidigoxigenin protein samples (A, B, and C) from different suppliers. All of the nanopore measurements were done with the same protocol described previously. As shown in Figure $4 \mathrm{a}$, the three binding curves reveal clear differences: the

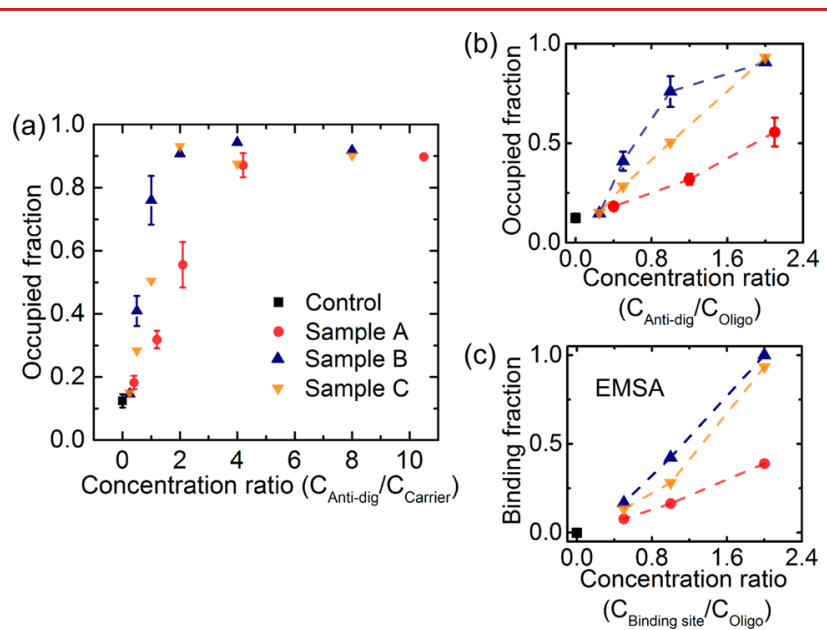

Figure 4. Comparison of active Anti-dig concentrations using three antibody samples (A, B, and C). (a) Binding curves obtained from three antidigoxigenin samples. The curve of sample $A$ is the same as in Figure $3 c$. The event numbers considered for sample B are 179, 218, $60,116,341$, and 507 for data points at the concentration ratio from 0.25 to 8 , respectively. The event numbers considered for sample $\mathrm{C}$ are $182,253,279,683,269$, and 301 for data points at the same concentration ratio from 0.25 to 8 , respectively (statistics in Supporting Information S8). (b) Zoomed-in concentration dependence of the occupied carrier fraction below the saturation point. Dashed lines are added as a guide to the eye. (c) Concentration dependence of the binding fraction obtained from EMSA.

saturation point of sample $A$ is at the concentration ratio of $\sim 4$, while the occupied fraction saturated at the ratio of $\sim 2$ for sample B and C. If the dissociation constant is assumed to be the same for all samples, the active protein concentration can be determined. Figure $4 \mathrm{~b}$ shows the increase in the zoomed in range before the saturated concentration ratio. Accordingly, sample $B$ and $C$ contain approximately twice more active antibodies which is consistent with the EMSA results in Figure 4c. EMSA gel images are in the Supporting Information, Figure S3.

The dynamic range for the high affinity binding protein systems tested here is approximately 1 order of magnitude. This lower limit is determined by the $5-10 \%$ of translocations that have false positive protein signal and the upper limit is the level at which the DNA is saturated with protein. The particular window of protein concentration tested can then be adjusted by changing the DNA carrier concentration (which we kept at 1.5 $\mathrm{nM}$ in these experiments). Since the translocation frequency of our DNA carriers is independent of the incubated protein concentration (Figure S6), higher concentrations could be used to achieve better statistics. To extend the dynamic range, a reduction in false positives by using shorter DNA lengths which show less folding is promising ${ }^{17}$ or by strategies to increase the bending rigidity of the DNA and therefore decrease the frequency of folds. Protein systems with lower binding affinities could also be investigated by employing chemical cross-linking strategies. All the latter techniques should greatly enhance the application range to other relevant protein systems. It is also important to note here that the single molecule nanopore approach lends itself to the possibility of multiplexed sensing 
which allows us to measure multiple proteins with a single nanopore. $^{18}$

In this work, we have shown that a combination of solid-state nanopores and designed DNA carriers can be used as a method for measuring nanomolar level of protein concentration. Several other studies of the translocation of DNA-protein complexes through solid-state nanopores have been used to qualitatively assess the presence of binding between DNA and proteins including the DNA-repair protein RecA, ${ }^{19}$ DNA antibodies ${ }^{20}$ and transcription factors. ${ }^{21}$ Other interesting work with similar ideas has been used to detect PNA and DNA targets where efforts have been made toward quantification. ${ }^{22}$ The results here show that quantitative estimates of protein concentration can be made using our DNA carrier analysis system by measuring the concentration dependence of the occupied carrier fraction. The working protein concentrations are at nanomolar range, and we use microfluidic chips with $\sim 10 \mu \mathrm{L}$ volume so the total amount of protein needed is only $10 \mathrm{fmol}$. The technique gives selectivity via protein binding sites and can be used for proteins in their native conformations without the requirement for any chemical modifications.

\section{ASSOCIATED CONTENT}

\section{S Supporting Information}

The Supporting Information is available free of charge on the ACS Publications website at DOI: 10.1021/acs.nanolett.6b00627.

Nanopore fabrication and size distribution, translocation data determination, EMSA and FP assay details, protein binding model, minimum sampling size discussion, and raw statistics of DNA translocation (PDF)

\section{AUTHOR INFORMATION}

\section{Corresponding Author}

*E-mail: ufk20@cam.ac.uk.

\section{Notes}

The authors declare no competing financial interest.

\section{ACKNOWLEDGMENTS}

The authors thank Howarth Lab, Oxford for providing the monovalent streptavidin sample, Yizhou Tan for the help with Labview programs, and Dr. Janet Kumita for the help with FP measurement. J. Kong acknowledges funding from Chinese Scholarship Council and Cambridge Trust. N.A.W.B. acknowledges funding from an EPSRC doctoral prize award and an ERC starting grant (Passmembrane 261101); U.F.K. acknowledges support from an ERC starting grant (Passmembrane 261101).

\section{REFERENCES}

(1) Walker, J. M. The protein protocols handbook; Springer Science \& Business Media, 1996; Vol. 1996.

(2) Domon, B.; Aebersold, R. Mass spectrometry and protein analysis. Science 2006, 312 (5771), 212-217.

(3) Hellman, L. M.; Fried, M. G. Electrophoretic mobility shift assay (EMSA) for detecting protein-nucleic acid interactions. Nat. Protoc. 2007, 2 (8), 1849-1861.

(4) Dandliker, W.; De Saussure, V. Fluorescence polarization in immunochemistry. Immunochemistry 1970, 7 (9), 799-828.

(5) (a) Engvall, E.; Perlmann, P. Enzyme-linked immunosorbent assay (ELISA) quantitative assay of immunoglobulin G. Immunochemistry 1971, 8 (9), 871-874. (b) Van Weemen, B.; Schuurs, A.
Immunoassay using antigen-enzyme conjugates. FEBS Lett. 1971, 15 (3), 232-236.

(6) (a) Rissin, D. M.; Kan, C. W.; Campbell, T. G.; Howes, S. C.; Fournier, D. R.; Song, L.; Piech, T.; Patel, P. P.; Chang, L.; Rivnak, A. J. Single-molecule enzyme-linked immunosorbent assay detects serum proteins at subfemtomolar concentrations. Nat. Biotechnol. 2010, 28 (6), 595-599. (b) Schubert, S. M.; Arendt, L. M.; Zhou, W.; Baig, S.; Walter, S. R.; Buchsbaum, R. J.; Kuperwasser, C.; Walt, D. R. Ultrasensitive protein detection via Single Molecule Arrays towards early stage cancer monitoring. Sci. Rep. 2015, 5, 11034.

(7) Zhang, Y.; Guo, Y.; Xianyu, Y.; Chen, W.; Zhao, Y.; Jiang, X. Nanomaterials for ultrasensitive protein detection. Adv. Mater. 2013, 25 (28), 3802-3819.

(8) (a) Tahvildari, R; Beamish, E.; Tabard-Cossa, V.; Godin, M. Integrating nanopore sensors within microfluidic channel arrays using controlled breakdown. Lab Chip 2015, 15 (6), 1407-1411. (b) Bell, N. A.; Thacker, V. V.; Hernández-Ainsa, S.; Fuentes-Perez, M. E.; Moreno-Herrero, F.; Liedl, T.; Keyser, U. F. Multiplexed ionic current sensing with glass nanopores. Lab Chip 2013, 13 (10), 1859-1862.

(9) Plesa, C.; Kowalczyk, S. W.; Zinsmeester, R.; Grosberg, A. Y.; Rabin, Y.; Dekker, C. Fast translocation of proteins through solid state nanopores. Nano Lett. 2013, 13 (2), 658-663.

(10) Larkin, J.; Henley, R. Y.; Muthukumar, M.; Rosenstein, J. K.; Wanunu, M. High-bandwidth protein analysis using solid-state nanopores. Biophys. J. 2014, 106 (3), 696-704.

(11) Li, W.; Bell, N. A.; Hernández-Ainsa, S.; Thacker, V. V.; Thackray, A. M.; Bujdoso, R.; Keyser, U. F. Single protein molecule detection by glass nanopores. ACS Nano 2013, 7 (5), 4129-4134.

(12) Bell, N. A.; Keyser, U. F. Specific protein detection using designed DNA carriers and nanopores. J. Am. Chem. Soc. 2015, 137 (5), 2035-2041.

(13) Howarth, M.; Chinnapen, D. J.; Gerrow, K.; Dorrestein, P. C.; Grandy, M. R.; Kelleher, N. L.; El-Husseini, A.; Ting, A. Y. A monovalent streptavidin with a single femtomolar biotin binding site. Nat. Methods 2006, 3 (4), 267-273.

(14) Rybak, J.-N.; Scheurer, S. B.; Neri, D.; Elia, G. Technical Brief Purification of biotinylated proteins on streptavidin resin: A protocol for quantitative elution. Proteomics 2004, 4, 2296-2299.

(15) (a) Lavén, M.; Velikyan, I.; Djodjic, M.; Ljung, J.; Berglund, O.; Markides, K.; Långström, B.; Wallenborg, S. Imaging of peptide adsorption to microfluidic channels in a plastic compact disc using a positron emitting radionuclide. Lab Chip 2005, 5 (7), 756-763. (b) Goebel-Stengel, M.; Stengel, A.; Taché, Y.; Reeve, J. R. The importance of using the optimal plasticware and glassware in studies involving peptides. Anal. Biochem. 2011, 414 (1), 38-46.

(16) (a) Daugherty, P. S.; Chen, G.; Olsen, M. J.; Iverson, B. L.; Georgiou, G. Antibody affinity maturation using bacterial surface display. Protein Eng., Des. Sel. 1998, 11 (9), 825-832. (b) Tetin, S. Y.; Swift, K. M.; Matayoshi, E. D. Measuring antibody affinity and performing immunoassay at the single molecule level. Anal. Biochem. 2002, 307 (1), 84-91.

(17) Bell, N. A.; Muthukumar, M.; Keyser, U. F. Translocation frequency of double-stranded DNA through a solid-state nanopore. Phys. Rev. E: Stat. Phys., Plasmas, Fluids, Relat. Interdiscip. Top. 2016, 93, 022401.

(18) Bell, N. A.; Keyser, U. F. Digitally encoded DNA nanostructures for multiplexed, single-molecule protein sensing with nanopores. Nat. Nanotechnol. 2016, DOI: 10.1038/nnano.2016.50.

(19) (a) Kowalczyk, S. W.; Hall, A. R.; Dekker, C. Detection of local protein structures along DNA using solid-state nanopores. Nano Lett. 2010, 10 (1), 324-328. (b) Smeets, R.; Kowalczyk, S.; Hall, A.; Dekker, N.; Dekker, C. Translocation of RecA-coated double-stranded DNA through solid-state nanopores. Nano Lett. 2009, 9 (9), 30893095.

(20) Plesa, C.; Ruitenberg, J.; Witteveen, M.; Dekker, C. Detection of Individual Proteins Bound along DNA Using Solid-State Nanopores. Nano Lett. 2015, 15 (5), 3153-3158.

(21) Squires, A.; Atas, E.; Meller, A. Nanopore sensing of individual transcription factors bound to DNA. Sci. Rep. 2015, 5, 11643. 
(22) (a) Carlsen, A. T.; Zahid, O. K.; Ruzicka, J. A.; Taylor, E. W.; Hall, A. R. Selective detection and quantification of modified DNA with solid-state nanopores. Nano Lett. 2014, 14 (10), 5488-5492. (b) Zahid, O. K.; Wang, F.; Ruzicka, J. A.; Taylor, E. W.; Hall, A. R. Sequence-specific recognition of microRNAs and other short nucleic acids with solid-state nanopores. Nano Lett. 2016, 16 (3), 2033-2039. 